\title{
A NEW PROOF OF A THEOREM ON QUASITRIANGULAR OPERATORS
}

GLENN R. LUECKE

\begin{abstract}
P. R. Halmos has given a proof of the equivalence of two definitions for quasitriangular operators. A short, elementary proof of this fact is given here.
\end{abstract}

In his paper Quasitriangular operators [1], Halmos proved the equivalence of the conditions $\left(\Delta_{0}\right)$ and $\left(\Delta_{2}\right)$ for operators $A$ on Hilbert space $H(\operatorname{dim} H=\infty)$. An operator satisfying $\left(\Delta_{0}\right)$ or $\left(\Delta_{2}\right)$ is called quasitriangular. The proof that $\left(\Delta_{0}\right)$ implies $\left(\Delta_{2}\right)$ is trivial. However, Halmos uses a three page proof to show that $\left(\Delta_{2}\right)$ implies $\left(\Delta_{0}\right)$. The following is a short and completely elementary proof of this fact.

Operator $A$ satisfies condition $\left(\Delta_{2}\right)$ if there exists a sequence $\left\{E_{n}\right\}$ of (orthogonal) projections of finite rank such that $E_{n} \rightarrow I$ (strong topology) and $\left\|A E_{n}-E_{n} A E_{n}\right\| \rightarrow 0$. Operator $A$ satisfies condition $\left(\Delta_{0}\right)$ if for every projection $P$ of finite rank and for every $\varepsilon>0$ there exists a finite rank projection $E \geqq P$ such that $\|A E-E A E\|<\varepsilon$.

TheORem (Halmos). If $A$ satisfies condition $\left(\Delta_{2}\right)$, then $A$ satisfies condition $\left(\Delta_{0}\right)$.

Proof. Use the notation above and let $Q_{n}$ be the projection on $E_{n}(N)$, where $N=P(H)$. Let $\frac{1}{2}>\delta>0$ be given. Since $\operatorname{dim} N<\infty$ and since $E_{n} g \rightarrow g$ for each $g \in H$, there exists $n_{0}$ such that for all $n \geqq n_{0},\left\|E_{n} g-g\right\|<\delta\|g\|$ for all $g \in N$. Let $n \geqq n_{0}$ and let $f \in E_{n}(N),\|f\|=1, f=E_{n} g, g \in N$. Then $\|g\| \leqq\left\|g-E_{n} g\right\|+\left\|E_{n} g\right\| \leqq \delta\|g\|+1$ so that $\|g\| \leqq(1-\delta)^{-1}$. Then

$$
\begin{aligned}
\left\|Q_{n} f-P f\right\| & =\|f-P f\|=\left\|E_{n} g-P E_{n} g\right\| \\
& \leqq\left\|E_{n} g-P g\right\|+\left\|P g-P E_{n} g\right\| \\
& \leqq\left\|E_{n} g-g\right\|+\|P\|\left\|g-E_{n} g\right\| \\
& \leqq(1+\|P\|) \cdot \delta\|g\| \leqq 2(1-\delta)^{-1} \delta<4 \delta .
\end{aligned}
$$

Furthermore if $f \in N,\|f\|=1$, then $P f=f$ and $Q_{n} f=E_{n} f$. Hence for $n \geqq n_{0}, \quad\left\|Q_{n} f-P f\right\|=\left\|E_{n} f-f\right\|<\delta$. Combining this with the previous

Received by the editors April 26, 1972 and, in revised form, June 9, 1972.

AMS (MOS) subject classifications (1970). Primary 47B99.

Key words and phrases. Operators, Hilbert space, quasitriangular operators.

(C) American Mathematical Society 1973 
statement we have that, for each $n \geqq n_{0},\left\|Q_{n} f-P f\right\|<4 \delta$ for all $f \in N \cup$ $E_{n}(N),\|f\|=1$. If $f \in\left(N \cup E_{n}(N)\right)^{\perp} \subseteq N^{\perp} \cap\left(E_{n}(N)\right)^{\perp}$, then $Q_{n} f=P f=0$. Taking the supremum (for each fixed $n \geqq n_{0}$ ) of $\left\|Q_{n} f-P f\right\|$ over all $\|f\|=1$, we obtain $\left\|Q_{n}-P\right\| \leqq 4 \delta$. Thus $\left\|Q_{n}-P\right\| \rightarrow 0$.

Define $O_{n}$ so that $E_{n}(H)=E_{n}(N) \oplus O_{n}$ and let $P_{n}$ be the projection on $N \oplus O_{n}$. Then $P_{n}$ has finite rank, $P_{n} \geqq P$ and, since $Q_{n}$ is the projection on $E_{n}(N), \quad\left\|E_{n}-P_{n}\right\|=\left\|Q_{n}-P\right\| \rightarrow 0$. Thus since $\left\|A E_{n}-E_{n} A E_{n}\right\| \rightarrow 0$ and $\left\|E_{n}-P_{n}\right\| \rightarrow 0$, we obtain $\left\|A P_{n}-P_{n} A P_{n}\right\| \rightarrow 0$. Therefore condition $\left(\Delta_{0}\right)$ holds.

\section{REFERENCE}

1. P. R. Halmos, Quasitriangular operators, Acta Sci. Math. (Szeged) 29 (1968), 283-293. MR 38 \#2627.

Department of Mathematics, Iowa State University, Ames, Iowa 50010 